\title{
BMJ Open Quality Junior doctor-led quality improvement project to improve safety and visibility of an interspecialty referral system
}

Dowan Kwon, ${ }^{1}$ Won Young Moon, ${ }^{2}$ Michelle Akhunbay-Fudge, ${ }^{3}$ Brandon Pieters, ${ }^{4}$ Jeni Pillai, ${ }^{5}$ Charlie Wilson, ${ }^{6}$ Jo Morrison (i) ${ }^{7}$

To cite: Kwon D, Moon WY, Akhunbay-Fudge $\mathrm{M}$, et al. Junior doctor-led quality improvement project to improve safety and visibility of an interspecialty referral system. BMJ Open Quality 2021;10:e001323. doi:10.1136/ bmjoq-2020-001323

- Additional supplemental material is published online only. To view, please visit the journal online (http://dx.doi.org/10. 1136/bmjoq-2020-001323).

Received 28 December 2020 Accepted 5 July 2021

Check for updates

(C) Author(s) (or their employer(s)) 2021. Re-use permitted under CC BY-NC. No commercial re-use. See rights and permissions. Published by BMJ.

For numbered affiliations see end of article.

Correspondence to

Dr Jo Morrison;

jo_morrison@doctors.org.uk

\section{ABSTRACT}

Interspecialty referrals for increasingly complex hospital inpatients are common and miscommunication often leads to delays in patient care. In a district general hospital, a web-based system generated an email referral, which lacked visibility and tracking/audit of the process, with no record generated automatically in paper inpatient notes or electronic patient records (EPR). We aimed to improve the visibility and safety of the interspecialty referral system. We canvassed stakeholders, informally and via an online satisfaction survey, collecting qualitative and quantitative data about attitudes to the existing system, generating ideas for change. We process mapped the system, identifying points of weakness. We adapted our EPR system, using a work-around solution, to develop a form that could be emailed from the EPR. This generated a permanent record within the EPR and an electronic record of the referral having been sent. We measured the visibility of referrals and responses within the EPR. We generated an online training 'how-to' video and reaudited stakeholder satisfaction.

There was a significant increase in the proportion of junior doctors satisfied or very satisfied with the interspecialty referral system (27/31 postintervention; $87.1 \%$ vs $26 / 55$ preintervention; 47\%; $p<0.0001$ ) and more believed that the visibility was adequate or very adequate (24/31 postintervention; $77.4 \%$ vs $9 / 55$ preintervention; $16.4 \%$; $p<0.0001)$. Visibility of referrals by project team members on the EPR increased from a baseline of $3.5 \%$ to $83.6 \%$ and the visibility of responses to these referrals on the EPR increased from $4.6 \%$ to $40.7 \%$. Qualitative feedback was excellent, hospital executive approval was gained and our work-around system spread to non-team members. We developed a more visible and reliable interspecialty referral system, adapting existing EPR capabilities, which was popular with users and led to cultural change in interspecialty referral responders. A formal EPR redesign, informed by our project, is in development.

\section{PROBLEM}

Somerset NHS Foundation Trust is a district general hospital in South-West England, delivering acute care to a local population of 340000 people and providing specialist services for a wider population of up to 800000 . The local population is relatively elderly with double the proportion of those aged over 65 and 80 years compared with the national average. ${ }^{1}$ All secondary healthcare is provided within the Trust, although some services are provided on a regional basis, requiring referral to university hospitals in different cities.

With increasing patient complexity and subspecialisation of clinical teams, more interspecialty referrals and opinions are required to optimise care of inpatients. A previous junior doctor quality improvement (QI) project developed a standardised paper interspecialty referral form. This had an adapted Situation/Background/Assessment/Recommendation (SBAR) format, $^{2}$ and contact details for the referring doctor. The paper form was subsequently transferred onto a web-based system. This generated an electronic interspecialty referral form, sending the completed form to a designated email address for each specialty (recipient), with a copy sent to the referrer. There were a number of concerns with the system:

1. The referral included only contact details for the doctor who generated the referral. With shift patterns and transfer of patients between teams, the referrer was often not available (or still responsible) for the patient when the recipient replied. This created a single point of failure, was a major safety concern for team members and the main driver for the project.

2. Others could not check a referral had been sent, as there was no record outside of the referrer's email account.

3 . The referring team were not sure, if no response to the referral was received, whether the referral had been made, received, responded to, or whether the delay was with the recipient responding to the referral.

4. None of the process was electronically auditable within the electronic patient record (EPR).

The web-based system had been introduced solely to replace a written paper form (which 
had a 'red top' to the page to highlight in paper notes and was hand-delivered to the receiving specialty) with an email delivery system. It would appear that at the time it was introduced auditing, visibility and standardisation of response were not considered. These were key concerns of the project team, but also significantly limited our ability to collect data, other than at an individual level, since no system collected or recorded the referrals outside of individual junior doctor email accounts, which are deleted after staff leave the Trust.

Our EPR system was introduced as an auditable electronic patient document management system and we did not have many of the functionalities of electronic health records often found in more holistic systems, such as Epic (https://www.epic.com/software). Previous junior doctor-led projects tried to address some of these issues, with one group successfully implementing a departmental-specific solution for referrals to the acute haematology-oncology team. They instigated a system that made referrals more visible by adding patient details to an inpatient list for the acute oncology team on the EPR system. This was therefore visible for all doctors to see, while patients remained on the list. However, this was a bespoke solution for a small team, with a lead involved in the QI project, lacked referrer details and visibility in the patients' electronic healthcare record and similar inpatient lists were not used universally by other teams, so remained limited in functionality.

We sought to develop an improved interspecialty referral system to improve auditability and visibility on the hospital EPR system. We hoped this would reduce delays in patient care and improve safety. Our SMART aim (specific, measurable, achievable, relevant and timebased) was to have at least $70 \%$ of the interspecialty referrals sent by our team members on the EPR by June 2019, to improve user satisfaction and referral visibility to $70 \%$ by June 2019 and to roll the system out Trust-wide by August 2019. As a secondary aim, we wanted to increase the visibility of responses on EPR by referral recipients.

\section{BACKGROUND}

Demands due to an increasingly elderly and comorbid population, combined with increased data burden from electronic health records, increase workload intensity for junior medical teams. ${ }^{3}$ As outlined by the UK Foundation Programme Office, ${ }^{4}$ this work is regularly performed by junior doctors during their foundation years (referred to as FY doctors for this article), the equivalent of an intern or postgraduate year 1 and year 2 in other healthcare structures. In an audit of 316 medical inpatients, 48 patients experienced a total of 53 delays, averaging an additional 1.8 days length of stay each, due to waiting for consultations or procedures from other specialties. ${ }^{5}$ Delays in referrals to procedure-heavy specialties were greatest, and miscommunication, due to a lack of a robust system, contributed to delays in patient care. Reducing delays in care and length of stay, especially for those with comorbidities and life-limiting illnesses, is a major aim of an NHS-wide project. ${ }^{6}$

Previous studies examined systems designed to improve quality of referrals from emergency medicine to hospital specialties. ${ }^{78}$ Others have looked at systems to reduce frustration from time to complete referrals and patient safety issues caused by poor interspecialty referrals. ${ }^{9}$ Many hospitals still rely heavily on paging devices (bleeps) for urgent front-of-house referrals. However, there is increasing adoption of innovative online referral systems with demonstrated benefit. ${ }^{10}{ }^{11}$ Nevertheless, despite their apparent superiority over traditional systems, online referral systems are not without weaknesses including: loss of real-time, two-way communication; need for audit/tracking of referrals to prevent patients getting 'lost in the system'; loss of accountability and need for robust handover; reduction in learning opportunities by discussing cases with specialists; and loss of human interaction and forming relationships with colleagues.

\section{MEASUREMENT}

We collected both qualitative and quantitative data to understand the problem and provide a baseline measurement. Qualitative baseline data on user views of the current interspecialty referral system were gathered using an online questionnaire. ${ }^{12}$ This was to determine the validity of our team's concerns, which were based on anecdotal incidents. This was sent to all junior doctors in the hospital.

The questions included:

1. Please rate your current level of satisfaction of the interspecialty referral system.

2. How adequate is the transparency/visibility of the interspecialty referral system? (between sending and receiving teams)

3. We plan to move the interspecialty referral system to EPR. Would this be of benefit?

Questions had multiple-choice answers on a 5-point Likert scale, ranging from very dissatisfied to very satisfied and very inadequate to very adequate for question numbers 1 and 2, respectively. Question number 3 had 'yes' and 'no' options. These questions were intended to be asked again at postintervention with no other variables altered to allow a like-for-like comparison.

Fifty-five junior doctors, all regular users of the interspecialty referral system, responded (out of 228 people; response rate $24.1 \%)$. Over a quarter $(n=15 ; 27.3 \%)$ were unsatisfied or very unsatisfied with the current interspecialty referral system and $37(67.3 \%)$ believed that the visibility of the referrals was either inadequate $(n=29$; $52.7 \%)$ or very inadequate $(\mathrm{n}=8 ; 14.6 \%)$ (online supplemental figure $1 \mathrm{~A}, \mathrm{~B})$. Almost all $(\mathrm{n}=52 ; 94.5 \%)$ of doctors believed moving the interspecialty referral system to a patient's EPR would be of benefit.

We also contacted the recipients of interspecialty referrals from 18 specialties to understand the downstream steps to the process, for example, who received the 


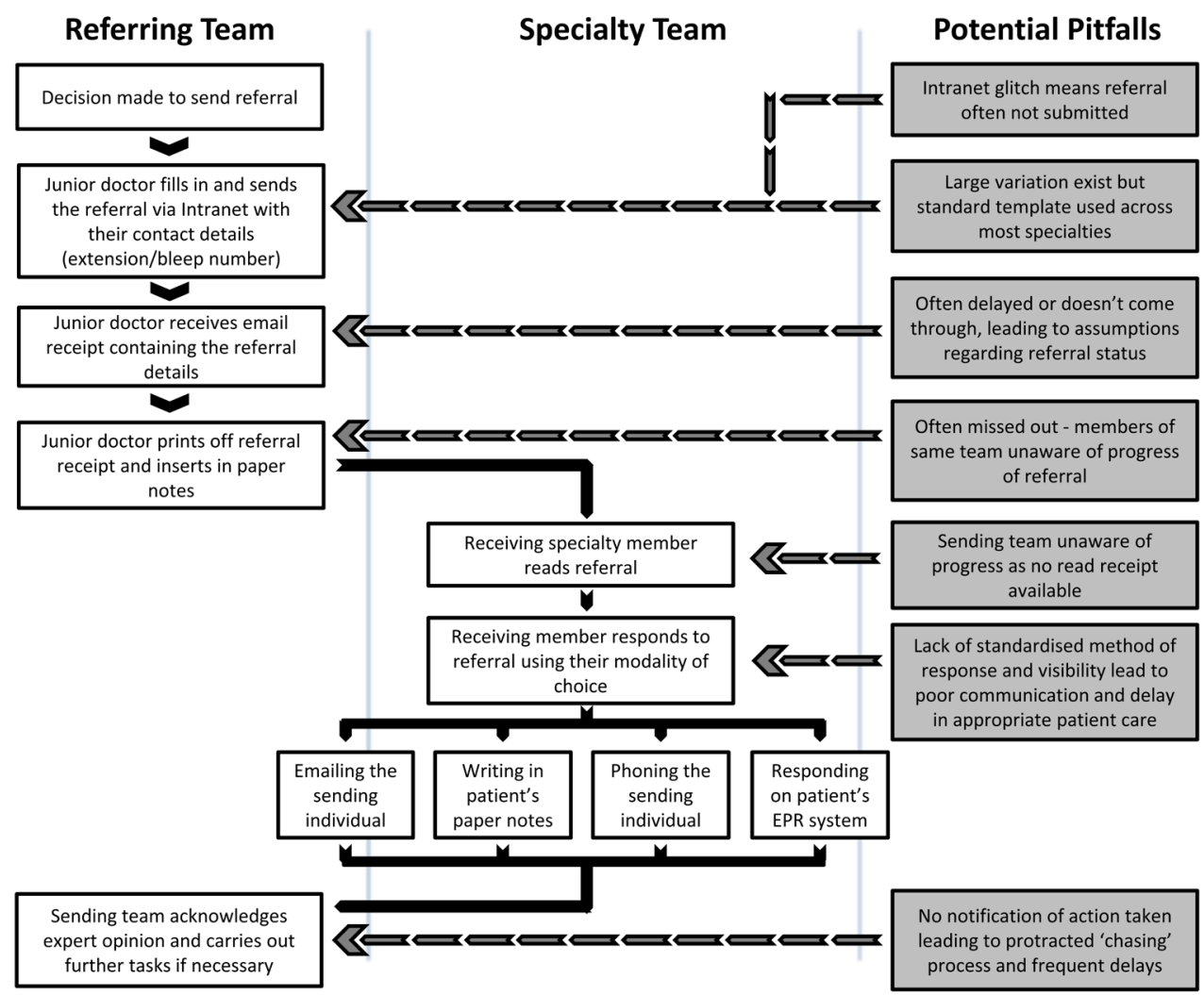

Figure 1 Process map of current interspecialty referral system and its pitfalls. EPR, electronic patient record.

interspecialty referral email, who actions the interspecialty referral and how responses were communicated to the referrer. This showed senior trainees, specialist associate doctors, nurse practitioners and specialist nurses were the direct recipients $(\mathrm{n}=12 ; 66.7 \%)$ and action takers $(n=16 ; 88.9 \%)$ of interspecialty referrals in most specialties, with senior clinician input. Thirteen specialties $(72.2 \%)$ used a mixed mode of communication to respond, with 16 specialties $(88.9 \%)$ seeing patients on the ward and writing in paper inpatient notes, although five specialties $(27.8 \%)$ commonly responding via email and nine $(50.0 \%)$ by phone.

Quantitative baseline data on interspecialty referral usage in the acute medical unit were captured over a period of 3 weeks. Due to the limited time period available for the project, a longer period of baseline data collection was not performed. Data were necessarily limited to those sent by the project team, due to the critical limitations of the web-based system (figure 1), whereby the referrer generates a form on a hospital intranet web page. This is forwarded to the email address of the referrer and a copy sent to the referring (often junior) doctor. There is no other record of this form stored or recorded and therefore no mechanism to interrogate referrals other than via individual email accounts. However, this quantified the methods of communication and timeframe of the actions taken. Of the 16 referrals sampled, none of the specific referrals, nor respective actions/responses, were visible on the EPR. One referral was never responded to. Of the remaining 15 referrals: $4(26.7 \%)$ resulted in a face-toface review, followed by actions/advice documented in paper notes; 6 (40\%) were resolved over the phone; and 5 $(33.3 \%)$ resulted in an email response or an email thread to an individual doctor. All but one referral had actions taken within 24 hours, with the exception being a secretary responding by email to acknowledge the referral and communicate that a follow-up would be arranged after the patient was discharged.

\section{DESIGN}

The QI project team consisted of six junior doctors at foundation training level, supported by consultants with QI methodology experience, as part of the Somerset NHS Foundation Trust FY1 junior doctor QI programme, between September 2018 and July 2019.

We sought to understand the problems and limitations of the current system, gathering opinions from those involved in sending and receiving interspecialty referrals. We used the data gathered from our baseline measurements to inform a process map of the current process and its pitfalls (figure 1). From this we generated a driver diagram to describe the aim, primary and secondary drivers and change ideas generated (figure 2).

From this we generated our 'ideal' system (online supplemental figure 2). We wanted referrals and responses to referrals to be made available in the patient's EPR, which was auditable and had functionality to send 


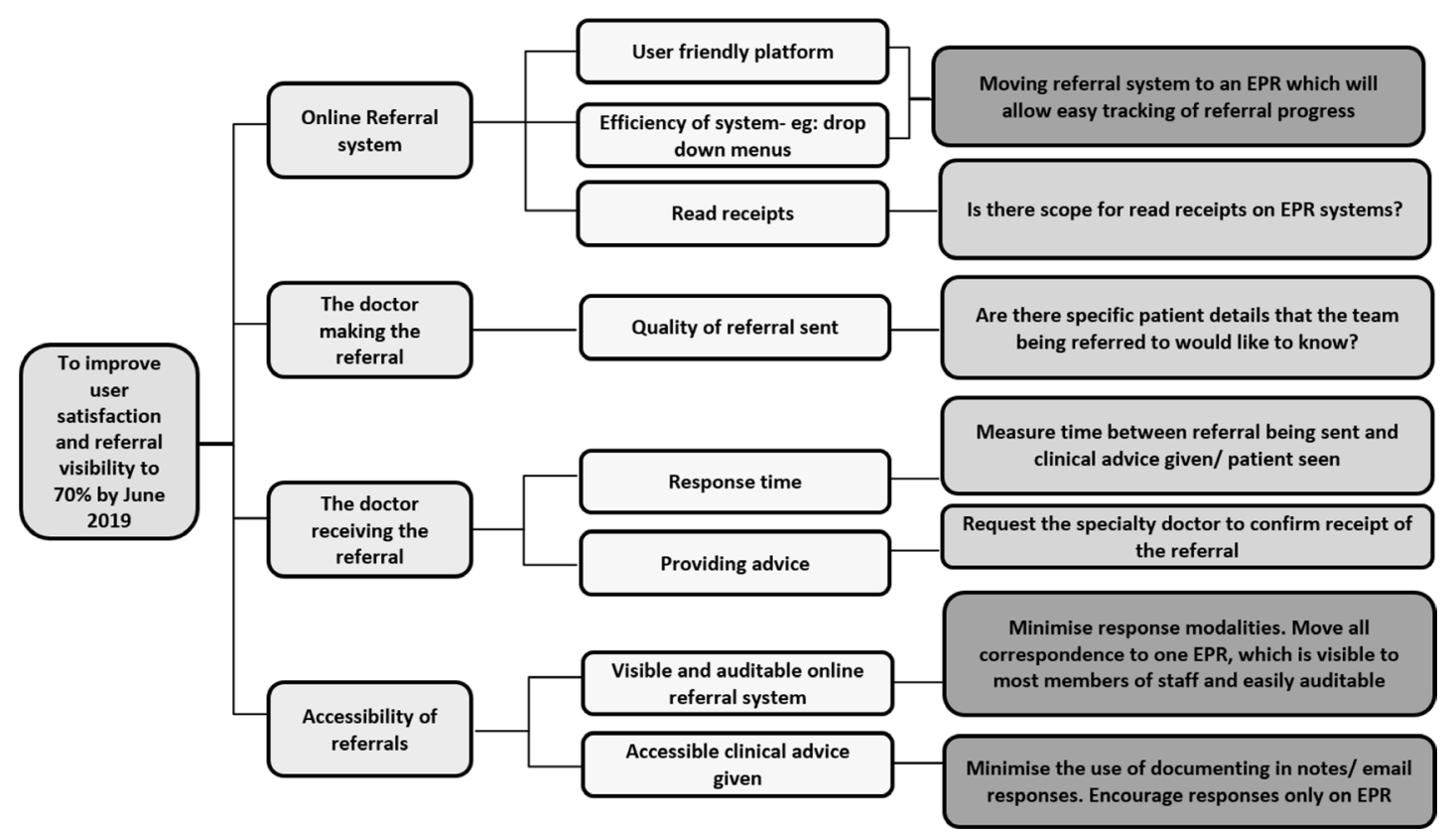

Figure 2 Driver diagram of project describing drivers and ideas for change in order to move towards an 'ideal' process (as described in online supplemental figure 2). EPR, electronic patient record. Change ideas in darker grey were targetted for this project.

documents as secure emails. It would also add clinical value and aid decision-making for future healthcare interactions, since most non-inpatient care was 'Paper-lite' and paper notes were not routinely available.

This solution was supported by most junior doctors, as demonstrated in our baseline survey. However, our ideal system would have involved a significant change to the hospital EPR (Epro by Bluewire Technology) with the generation of a new form and read-receipt capabilities. ${ }^{13}$ Due to the EPR system being owned outside of the Trust, this was not possible at that stage. We therefore developed a work-around solution within the existing capabilities, without a formal digital transformation. The team met every 4-8weeks to review the database and PlanDo-Study-Act (PDSA) cycles, with regular correspondence with the information technology (IT) team and trust digital strategy team (Digital Hub) for advice and support.

\section{STRATEGY}

We collected data on all interspecialty referrals sent by team members, searching email folders for copy emails generated by the web-based system and copies sent from the EPR. The data points were analysed using Life QI, ${ }^{14}$ an online QI project and methodology platform. Due to the lack of reliable visibility of referrals within either patient notes or the EPR, we were limited in our data collection, unable to sample data from a wider cohort of referrers, or a random sample of patient records, highlighting significant issues with the system.

\section{PDSA cycle 1 (22 November 2018)}

We explored with the IT team whether a read-receipt could be automatically generated by the web-based interspecialty referral form/email. Unfortunately, this was not possible. We therefore took a low-technology approach and added a standard text request as a 'cut and paste' to the bottom of each interspecialty referral, within the existing web-based email system. This briefly explained our QI project and requested the recipient reply to acknowledge receipt of referral.

\section{PDSA cycle 2 (1 April 2019)}

The second change idea was to generate the interspecialty referral form as a letter on the EPR. This would be auditable and visible for all, even after discharge. We created an interspecialty referral form template as an auto-text within the EPR using the existing form's SBAR format. This letter could be emailed to the referral recipient and sender (auditable within EPR). We collected specialtyspecific referral email addresses and added these to the EPR database, with the help of the Digital Hub team. We monitored compliance with this by auditing referrals sent by project team members only. Sending interspecialty referrals through EPR was not more complex or time consuming than the existing web-based system.

\section{PDSA cycle 3 (1 May 2019)}

As referring via the EPR became routine for team members, we noticed responders were more likely to respond via the EPR. We therefore added a stock phrase to the standard interspecialty referral auto-text, asking 
A

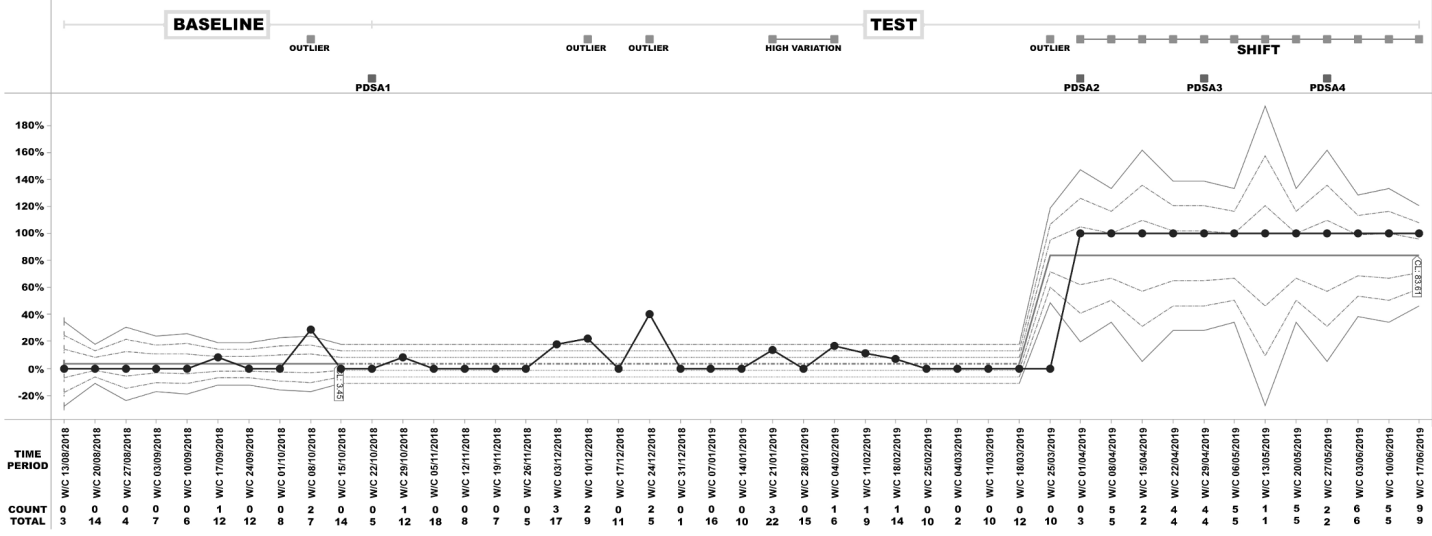

B

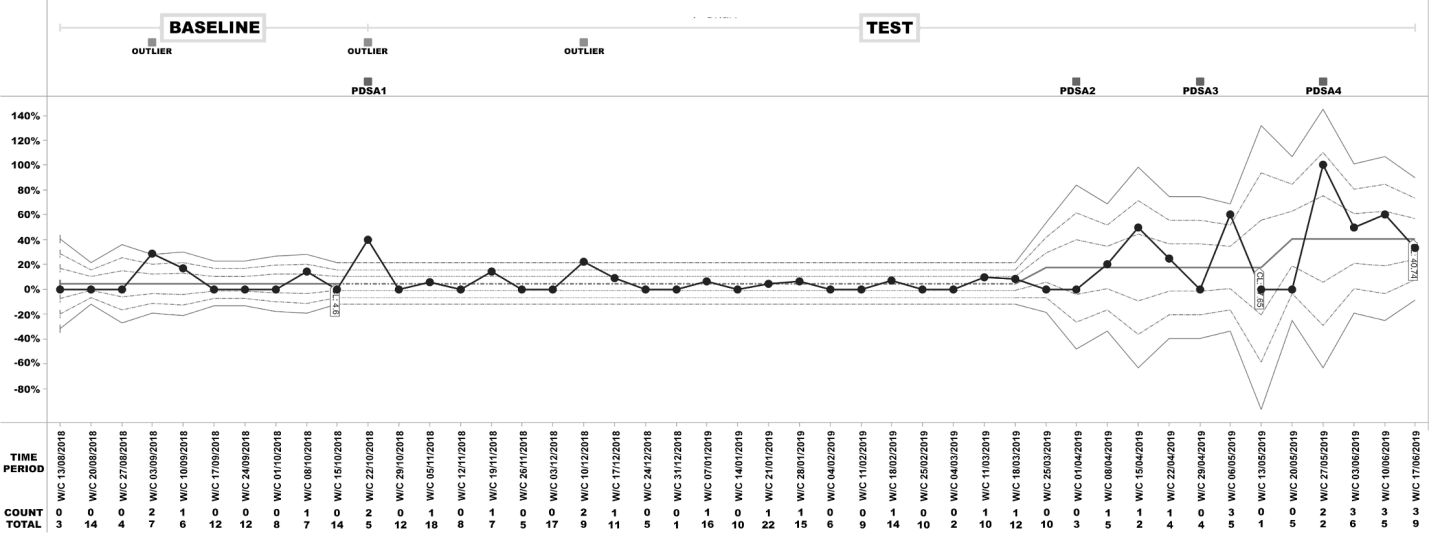

Figure 3 Run charts of visibility of (A) referrals and (B) responses to referrals in the electronic patient record of those referrals sent by the group members during the project period. PDSA, Plan-Do-Study-Act.

for a record of the response to be added to the EPR, explaining the aim to improve visibility of referrals and responses.

\section{PDSA cycle 4 (12 July 2019)}

To spread and sustain the system outside of the team, and in preparation for the August junior doctor changeover, we designed a video tutorial for how to use the new interspecialty referral system (online supplemental data video). We attached an explanation of the project and included an electronic postintervention survey.

\section{RESULTS}

In PDSA cycle 1, no one responded with an email to acknowledge receipt of referral other than a couple of responses asking us to stop bothering them, although this did confirm receipt.

In PDSA cycle 2, the visibility of referrals on the EPR system shifted significantly as the junior doctor members of the QI project team began to adopt the new system into their daily practice. Consequently, the visibility of the referrals from the team members increased from a preintervention baseline of $3.5 \%$ to $83.6 \%$ (figure $3 \mathrm{~A}$ ). Other junior doctors also started to adopt this method of sending referrals widely, with organic spread of the system throughout the junior doctor cohort. Unfortunately, the rate of other junior doctors using the new system was not measurable due to the very significant limitations of the existing web-based referral system, which recorded no data, and the EPR system, which could only audit editing/sending of emails within the context of an individual patient.

In PDSA cycle 3, despite our prediction that people would be resistant to change, there was a gradual increase in percentage of responses on the EPR (figure 3B). Overall, there was no statistically significant shift or trend. However, the centre line value increased from $4.6 \%$ to $17.65 \%$ after the second PDSA cycle and to $40.74 \%$ following the third PDSA cycle. The lack of significance was in part due to the timescale of the project and limited number of data points, as at this stage junior doctors rotated to different posts/hospitals.

PDSA 4 was received enthusiastically by other junior doctors, many of whom began to use this system having seen others use it or after seeing the tutorial video. There was no significant difference in the distributions of grade of the respondents between preintervention and postintervention survey $\left(\chi^{2}\right.$ test for trend; $\left.\mathrm{p}=0.17\right)$. There was a significant increase in the proportion of junior doctors who were satisfied/very satisfied with the new system 
compared with the web-based referral system $(27 / 31$; $87.1 \%$ vs $26 / 55 ; 47.3 \%$; $<0.0001)$. Furthermore, a higher proportion of junior doctors believed the visibility of the new system was adequate/very adequate compared with the existing system $(24 / 31 ; 77.4 \%$ vs $9 / 55 ; 16.4 \%$; $\mathrm{p}<0.0001$ ) (online supplemental figure 1A,B).

Qualitative feedback was overwhelmingly positive and included:

I've used this extensively, the only issue is that some people still aren't using it, so the more people that can switch to the new system the better!

It's so great! Saved work being repeated when people change shift and means everyone is aware what information was shared. Brilliant.

This project was presented to the hospital executive team at the FY1 QI programme presentation day. There was widespread approval and enthusiasm for the new system, which was recognised as being significantly safer. We were instructed to introduce this to the incoming junior doctor cohort by the chief medical officer.

However, the digital safety lead and chief clinical information officer required the new system to go through IT safety testing, despite acknowledging that the new system was a significant improvement on existing web-based system. We worked with the Digital Hub team to develop a formal EPR-based system, which would incorporate all of the aspects we had identified as our 'ideal' system. This has been delayed due to licensing issues and the COVID-19 pandemic placing more urgent demands on the IT teams. The new system is now ready for testing and will hopefully be introduced as standard to the hospital within the next 6 months. In the mean time, our EPR system has continued to be used by those doctors who stayed in the hospital and has organically spread to incoming doctors, despite lack of formal adoption.

\section{LESSONS AND LIMITATIONS}

Due to the significant limitations of our existing web-based referral system, we were only able to collect data on mode of referral for the team members, rather than on a wider scale. This is an inherent and unavoidable bias of the data we were able to collect, since the team were highly likely to use the new system. It also limited the amount of data we were able to collect for baseline data and demonstration of change, since there was no system that recorded referrals other than individual doctor Trust email accounts. However, this limitation in our ability to perform wider data collection also emphasised the need for system change. We learnt that delivering change via email 'nudges' was wholly ineffective.

Although we noticed an organic uptake of our new referral system by junior doctors outside of the QI project team, we could not formally measure this, as the EPR lacked auditability function. This is an add-on function, which has been requested specifically to the digital team going forward.

Notably, after increasing the visibility of the referral content, we observed an increase in the visibility of referral responses especially following PDSA cycle 2. A larger sample size for a longer duration towards the end of the data collection period may have resulted in a significant trend or a shift, which is a limitation of the project, as data collection stopped when the FY1 team moved on.

The major limitation to our project was adaptability of existing IT systems and the pace of change possible, due to need to mitigate risks, especially when IT systems are delivered by external partners.

Although we were not able to formally introduce the changes on a hospital-wide scale, we were able to adapt an existing EPR system and demonstrate a need for formal system change. Use of our work-around solution spread widely through junior staff working at that time, and has persisted, despite several rotations through the hospital, supporting the positive responses to our postintervention survey. Spread and sustainability of change is often a major hurdle in any QI project and this suggests it will not be a concern once a new system, based on our 'ideal' system, is formally introduced.

Ideally, the Trust would employ a holistic patient healthcare record system, such as Epic, that will do all of the elements we identified as needed in an ideal system. Unfortunately, such holistic systems, or add-ons for our current system, come at considerable cost and are not deemed affordable in many healthcare settings, including our own.

\section{CONCLUSION}

We observed, anecdotally, that miscommunication between specialties contributed to unnecessary delay in patient care, prolonged length of inpatient stay and increased workload intensity for staff members. We demonstrated ease of communication and user satisfaction were improved by adapting an existing EPR using step-by-step QI methodology. This project, carried out by six of the most junior doctors, highlights that anyone can make a difference.

Further work is in progress, as the hospital board has invested to make a Trust-wide change to implement a more permanent solution, based on our project.

\section{Author affiliations}

${ }^{1}$ South Bristol Academy/Undergraduate Education Department, Trust Services, Bristol Royal Infirmary, Bristol, UK

${ }^{2}$ Department of Ophthalmology, Royal Berkshire NHS Foundation Trust, Reading, UK ${ }^{3}$ Department of Acute Internal Medicine, Wexham Park Hospital, Slough, UK

${ }^{4}$ Musgrove Park Academy, Somerset NHS Foundation Trust, Taunton, UK

${ }^{5}$ Department of Psychiatry, Homerton University Hospital, East London Foundation Trust, London, UK

${ }^{6}$ Department of Anaesthetics, Somerset NHS Foundation Trust, Taunton, UK ${ }^{7}$ Department of Gynaecological Oncology, Somerset NHS Foundation Trust, Taunton, UK

Twitter Jo Morrison @DrJoMorrison1

Acknowledgements The project was performed as part of a wider FY1 Q programme by FY1s and senior mentors volunteering in their own time, out of hours. We would like to thank: Somerset NHS Foundation Trust Digital Hub and IT teams, especially Alice Sweeting, for their support, advice and help with uploading the specialty-specific emails to the EPR; Shanice Cox, medical PA, for her help with the EPR referral templates, generating auto-texts and assigning these to junior doctors; Dr Jo Botten, for coleading the junior doctor (FY1) Ql programme with JM; 
Dr Chris Knight for his mentorship; Somerset NHS Foundation Trust Executive Team for their continued support for the FY1 QI programme.

Contributors DK, WYM, MAF, BP, JP, CW and JM contributed equally to the study idea, design, data collection and analysis. Additional data analysis, statistics and writing of the article were performed by DK and JM. DK, WYM, MAF, BP, JP, CW and JM approved the final text.

Funding The authors have not declared a specific grant for this research from any funding agency in the public, commercial or not-for-profit sectors.

Competing interests None declared.

Patient and public involvement statement Patients were not involved in the design or implementation of this study.

Patient consent for publication Not required.

Provenance and peer review Not commissioned; externally peer reviewed.

Data availability statement All data relevant to the study are included in the article or uploaded as supplementary.

Supplemental material This content has been supplied by the author(s). It has not been vetted by BMJ Publishing Group Limited (BMJ) and may not have been peer-reviewed. Any opinions or recommendations discussed are solely those of the author(s) and are not endorsed by BMJ. BMJ disclaims all liability and responsibility arising from any reliance placed on the content. Where the content includes any translated material, BMJ does not warrant the accuracy and reliability of the translations (including but not limited to local regulations, clinical guidelines, terminology, drug names and drug dosages), and is not responsible for any error and/or omissions arising from translation and adaptation or otherwise.

Open access This is an open access article distributed in accordance with the Creative Commons Attribution Non Commercial (CC BY-NC 4.0) license, which permits others to distribute, remix, adapt, build upon this work non-commercially, and license their derivative works on different terms, provided the original work is properly cited, appropriate credit is given, any changes made indicated, and the use is non-commercial. See: http://creativecommons.org/licenses/by-nc/4.0/.

ORCID iD

Jo Morrison http://orcid.org/0000-0003-0000-520X

\section{REFERENCES}

1 Age group profiles for Somerset, 2020. Available: http://www. somersetintelligence.org.uk/age-group-profiles-for-somerset.pdf

2 Institute for Healthcare Improvement. SBAR tool: SituationBackground-Assessment-Recommendation Cambridge, Massachusetts. USA: Institute for Healthcare Improvement, 2021. http://www.ihi.org/resources/Pages/Tools/SBARToolkit.aspx

3 Clark AV, LoPresti CM, Smith TI. Trends in inpatient admission comorbidity and electronic health data: implications for resident workload intensity. J Hosp Med 2018;13:570-2.

4 UK Foundation Programme Office. The UK Foundation programme curriculum.: UKFoundationProgrammeOffice, 2016. Available: https:// content.hee.nhs.uk/curriculum/bibliography.html [Accessed 20 Nov 2020].

5 Rahman AS, Shi S, Meza PK, et al. Waiting it out: consultation delays prolong in-patient length of stay. Postgrad Med J 2019;95:1-5.

6 NHS Improvement. Guide to reducing long Hospital stays 2019. Available: https://improvement.nhs.uk/resources/guide-reducinglong-hospital-stays/\#h2-find-out-more [Accessed 19 Mar 2019].

7 Reid C, Moorthy C, Forshaw K. Referral patterns: an audit into referral practice among doctors in emergency medicine. Emerg Med J 2005;22:355-8.

8 Lawrence S, Spencer LM, Sinnott M, et al. It takes two to tango: improving patient referrals from the emergency department to inpatient clinicians. Ochsner J 2015;15:149-53.

9 Cathcart J, Cowan N, Tully V. Referral finder: saving time and improving the quality of in-hospital referrals. BMJ Qual Improv Rep 2016;5:u209356.w3951.

10 Shephard E, Stockdale C, May F, et al. E-referrals: improving the routine interspecialty inpatient referral system. BMJ Open Qual 2018;7:e000249.

11 Ziff OJ, Routledge E, Turner C, et al. Modernising inpatient referral systems: switching from 'on call' to 'online'. Postgrad Med J 2019;95:292.

12 SurveyMonkey [Internet]. San Mateo, California, USA: SurveyMonkey Inc. Available: https://www.surveymonkey.com [Accessed 4 Dec 2020].

13 Epro [Internet]. Bristol, UK: Epro. Available: https://www.epro.com [Accessed 9 May 2021].

14 Life QI [Internet]. Exeter, UK: Life QI. Available: https://uk. lifeqisystem.com [Accessed 4 Dec 2020]. 